\title{
Bariatric surgery outcomes: a single-center study in the United Arab Emirates
}

This article was published in the following Dove Press journal:

Diabetes, Metabolic Syndrome and Obesity:Targets and Therapy

18 September 2015

Number of times this article has been viewed

\section{Salah Abusnana' \\ Sarah Abdi' \\ Brigette Tagure' \\ Murtada Elbagir' \\ Almantas Maleckas²}

'Rashid Center for Diabetes and Research, Ministry of Health, Ajman, United Arab Emirates; ${ }^{2}$ Kaunas University of Medicine, Kaunas, Lithuania
Correspondence: Salah Abusnana Rashid Center for Diabetes and Research, Ministry of Health, PO Box 21499, Ajman, United Arab Emirates Tel $+97|67| 47344$

Fax +97I 67434547

Email salah.abusnana@rcdr.ae
Background: Bariatric surgery has become an attractive treatment for severe obesity over the last decade, due to its impacts on weight loss and remission of type 2 diabetes and metabolic syndrome. In the United Arab Emirates, a country where the rate of obesity is dramatically increasing bariatric surgery has gained popularity in recent years; however, published data on its outcomes in the Emirati population are lacking.

Methods: We retrospectively reviewed the medical records of 95 patients who underwent bariatric surgery (ie, laparoscopic Roux-en-Y gastric bypass [RYGB] or laparoscopic sleeve gastrectomy) at the Rashid Center for Diabetes and Research in Ajman, United Arab Emirates. Weight outcomes and metabolic marker data were abstracted at baseline and at 3, 6, and 12 months postoperatively.

Results: Laparoscopic RYGB was the main procedure performed by our bariatric unit. All variables demonstrated postoperative improvement. An average excess weight loss of $68 \%$ was observed at 12 months. Fat mass was the body component that decreased the most, with an average reduction of $46 \%$. Additionally, lipid profiles were significantly different $(P<0.01)$ at 12 months, with triglyceride levels improving by $27 \%$ and low-density lipoprotein levels improving by $21 \%$. Similarly, glycated hemoglobin $\left(\mathrm{HbA}_{1 \mathrm{c}}\right)$ levels decreased significantly $(P<0.001)$ in patients with type 2 diabetes, with an average reduction of $73 \%$.

Conclusion: Our results show that a substantial short-term reduction in weight and significant improvements in metabolic markers followed bariatric surgery in severely obese Emirati patients. Our results are consistent with the outcomes of other internationally published studies. Additional studies are warranted to determine whether the favorable impacts of bariatric surgery can be sustained over the long term.

Keywords: laparoscopic RYGB, laparoscopic sleeve gastrectomy, lipid profile, type 2 diabetes

\section{Introduction}

Obesity and its comorbidities, including diabetes and cardiovascular disease, are considered to have serious impacts that increase the public health burden worldwide. ${ }^{1}$ The same is true in the United Arab Emirates (UAE), a country that has encountered a dramatic transformation in its economy and society since the first exports of oil in 1962. The UAE has one of the highest proportions of overweight and obese adults aged 15 years and older in the Eastern Mediterranean region. ${ }^{2}$ Two non-communicable diseases that are attributable to obesity, ie, diabetes and cardiovascular disease, have been identified by the local Ministry of Health as the leading cause of mortality in the UAE. ${ }^{3}$ Beyond its impact on mortality, obesity has been suggested to be the main driver of increasing health care costs in many countries. ${ }^{4}$ As body mass index (BMI) 
increases, the associated direct health care costs and losses of productivity increase correspondingly. ${ }^{4}$ In the UAE, the costs associated with caring for diabetes, one of the metabolic consequences of obesity, are high (\$1,605 per diabetic patient) and those associated with the management of its complications are significantly higher $(\$ 5,645$ per diabetic patient $){ }^{5}$

Many treatment options exist for the management of obesity, including both nonsurgical and surgical treatments. Dietary therapy, physical activity, behavior therapy, pharmacotherapy, or combinations of these strategies are considered effective nonsurgical weight loss interventions. ${ }^{6}$ However, in patients with severe obesity (BMI $\geq 40 \mathrm{~kg} / \mathrm{m}^{2}$ ) or with $\mathrm{BMI} \geq 35 \mathrm{~kg} / \mathrm{m}^{2}$ and associated comorbidities, and for whom these strategies have failed to produce the desired weight loss, a surgical approach is suggested. ${ }^{6}$ Moreover, bariatric surgery has been shown to be superior to nonsurgical approaches in terms of weight loss and remission of type 2 diabetes and metabolic syndrome over both the short term and long term. ${ }^{7,8}$ Indeed, bariatric surgery is considered an appealing treatment for severe obesity worldwide. In the last decade, the number of bariatric surgeries performed worldwide has increased substantially, doubling between 2003 and 2008 from 143,301 to 344,221 procedures per year. ${ }^{9}$ Similarly, in the UAE, bariatric surgery has become a favored treatment for severe obesity in recent years; 1,963 such surgeries were performed in 2011 alone. ${ }^{10}$ The two most commonly performed bariatric procedures in the UAE and internationally are the laparoscopic Roux-en-Y gastric bypass (RYGB) and laparoscopic sleeve gastrectomy. ${ }^{10}$

A recent report comparing bariatric outcome in the UAE with that in American College of Surgeons National Surgical Quality Improvement Program hospitals found that outcomes were similar for most postoperative variables. ${ }^{11}$ The report focused on the safety of bariatric surgery in terms of postoperative complications; changes in weight and metabolic markers were not reported. Despite this similarity in the quality of health care for bariatric patients, it would be inaccurate to presume that the same similarities would exist in terms of metabolic outcomes, because other factors such as ethnicity might affect the outcome of bariatric surgery. ${ }^{12}$ Despite the marked increase in bariatric surgery in the Arab Gulf region over the last decade, no data have been published on its metabolic outcomes in adults with this ethnic background. To the best of our knowledge, this is the first study to report bariatric metabolic outcomes in an Emirati population. This retrospective study reports results obtained by the bariatric program at the Rashid Center for Diabetes and Research (RCDR) in Ajman, UAE, and describes the preoperative and postoperative care programs, patient characteristics, and short-term postoperative outcomes (weight and comorbidity parameters). The results obtained here were compared with those obtained internationally.

\section{Materials and methods Bariatric unit at RCDR}

The bariatric surgery unit at RCDR adopted three main objectives when it was first established: first, to develop the bariatric surgery pathway based on recently developed guidelines; second, to set up a multidisciplinary team (including an endocrinologist, bariatric surgeon, nurse, dietitian, anesthesiologist, cardiologist, and psychiatrist) to provide comprehensive care for patients before and after surgery; and third, to set up a long-term follow-up plan for all patients who have undergone bariatric surgery at RCDR.

\section{Subjects}

All patients offered surgical intervention were at least 18 years old and had been referred to the bariatric unit at RCRD. Patients included in this study were those with complete data on demographics, weight, and clinical outcomes, and eligible for the 1-year follow-up. All patients provided written informed consent prior to the surgical procedure after a thorough discussion with the multidisciplinary team during the preoperative care program. Either laparoscopic RYGB or laparoscopic sleeve gastrectomy was offered, depending on the individualized therapy goals, patient preferences, and risk assessments. Most patients underwent laparoscopic RYGB; 12-month follow-ups were completed in February 2014. The study was approved by the ethical review committee at the RCDR, Ajman.

\section{Preoperative care}

As a prerequisite for enrolling in the preoperative program, all potential candidates attended a group seminar describing the benefits and risks of bariatric surgery. The entire multidisciplinary bariatric team was involved in the educational session. Investigative individual consultations with the multidisciplinary team then followed, in which all potential candidates were screened for inclusion/exclusion criteria according to the American Society for Metabolic and Bariatric Surgery (ASMBS) guidelines. ${ }^{13}$ The ASMBS inclusion criteria for bariatric surgery candidates are as follows: BMI $\geq 40 \mathrm{~kg} / \mathrm{m}^{2}$ with no comorbidities or BMI $\geq 35 \mathrm{~kg} / \mathrm{m}^{2}$ with obesity-associated comorbidities, including type 2 diabetes, hypertension, hyperlipidemia, obstructive sleep apnea, and nonalcoholic fatty liver disease; failure in previous 
nonsurgical attempts to lose weight; and the expectation that the patient will comply with postoperative care (follow-up visits with the health care team, and compliance with dietary supplements postoperatively and any instructions regarding the surgical procedure). Patients were excluded from the bariatric program based on ASMBS exclusion criteria, which are as follows: current drug or alcohol abuse; reversible endocrine or other disorder that can cause obesity; uncontrolled/ severe psychiatric illness; and lack of comprehension of the risks/benefits/expected outcomes/lifestyle changes associated with bariatric surgery. If eligible for surgical intervention, patients were referred to the preoperative program. This included two to three scheduled visits with the endocrinologist, bariatric nurse, dietitian, cardiologist, anesthesiologist, and psychologist; during these visits, detailed assessments of medical history and continuous anthropometric, biochemical, and dietary assessments were conducted. One week before the surgery, an educational session was conducted to inform the patients regarding the admission process, the day of surgery, and post-surgery instructions (Figure 1).

\section{Postoperative care}

Surgeries were performed by one specialized bariatric surgeon at the adjacent Sheikh Khalifa Hospital, Abu Dhabi, under supervision by the bariatric surgery unit at RCDR. Patients' length of stay in the hospital depended on the type of surgery; the patients were discharged by the surgeon, based on their ability to drink $1.5 \mathrm{~L}$ of fluid over 24 hours without pain, nausea, vomiting, or other discomfort. Patients were then scheduled for a telephone call with the bariatric nurse 5 days after surgery and a clinic visit 10 days after surgery for suture/ staple removal by the surgeon. This was followed by visits at 1, 3, 6, and 12 months, which included determination of outcome, reassessment by the endocrinologist, bariatric nurse, and dietitian, reinforcement of nutrition guidelines, and advice regarding vitamin/mineral supplement intake, if indicated.

\section{Outcome measures}

The following information was abstracted from patient medical records: sociodemographics, including age and sex; anthropometric data, including weight and height,

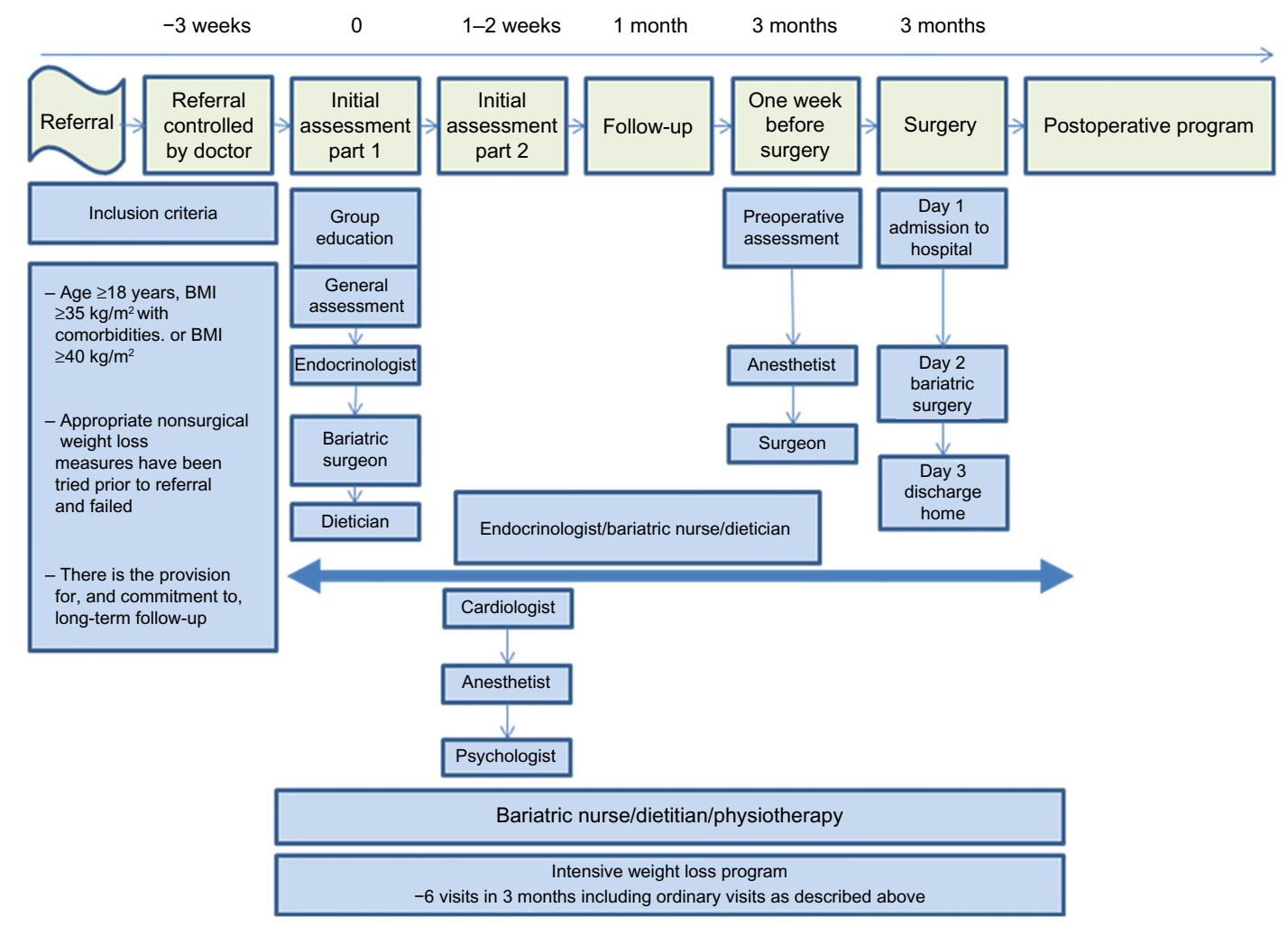

Figure I Preoperative bariatric program at Rashid Center for Diabetes and Research.

Notes: All candidates attended a group education seminar prior to the program, in which the entire multidisciplinary bariatric team was involved. Investigative individual consultations with the multidisciplinary team then followed, in which all potential candidates were screened for inclusion/exclusion criteria according to the American Society for Metabolic and Bariatric Surgery guidelines. If eligible for surgical intervention, patients were referred to the preoperative program. This program included two to three scheduled visits with the endocrinologist, bariatric nurse, dietitian, cardiologist, anesthesiologist, and psychologist, during which detailed assessments of medical history and continuous anthropometric, biochemical, and dietary assessments were conducted. One week before surgery, an educational session was conducted to inform the patients regarding the admission process, the day of surgery and post-surgery instructions.

Abbreviation: BMI, body mass index. 
as measured by the bariatric nurse for all patients, BMI (calculated as weight $[\mathrm{kg}] /$ height $\left[\mathrm{m}^{2}\right]$ ), body composition analysis, based on fat mass $(\mathrm{kg})$, muscle mass $(\mathrm{kg})$, water mass (kg), as measured by bioelectrical impedance using an InBody 230 equipment (Biospace, Korea) in accordance with standard operating procedures; metabolic marker outcomes, including blood pressure, as measured using a standard sphygmomanometer equipped with an appropriate cuff size, fasting blood glucose, insulin, and glycated hemoglobin $\left(\mathrm{HbA}_{1 \mathrm{c}}\right)$ levels, lipid profile (low-density lipoprotein, high-density lipoprotein, triglycerides, and total cholesterol), liver profile (alkaline phosphatase, alanine transaminase, aspartate transaminase, and gamma-glutamyl transferase), and albumin (all laboratory tests were conducted using a Roche Diagnostics machine, Japan); and any medication used by patients with type 2 diabetes, including biguanides (metformin), sulfonylureas (gliclazide), glucagon-like peptide-1 (GLP-1) agonist therapy (exenatide), antihypertensive medication (angiotensin receptor blockers), and medications for hyperlipidemia (statins).

\section{Definitions}

Percentage of excess weight loss (\% EWL) was calculated by dividing the amount of weight loss after surgery by the amount of presurgery excess body weight (calculated as that excess to yield a BMI of $25 \mathrm{~kg} / \mathrm{m}^{2}$ ) and multiplied by $100 .{ }^{14}$ Type 2 diabetes was defined using the American Diabetes Association definition of type 2 diabetes $\left(\mathrm{HbA}_{1 \mathrm{c}} \geq 6.5 \%\right.$ or fasting blood glucose $\geq 7 \mathrm{mmol} / \mathrm{L}$ ). Complete remission of type 2 diabetes was defined using the American Diabetes Association definition: " $\mathrm{HbA}_{1 \mathrm{c}}$ in the normal range and fasting blood glucose $<100 \mathrm{mg} / \mathrm{dL}$ for at least 1 year duration in the absence of active pharmacological agent or ongoing procedure". ${ }^{15}$ Fat free mass $(\mathrm{kg})$ was calculated by subtracting fat mass $(\mathrm{kg})$ from total weight $(\mathrm{kg})$.

All data were abstracted at baseline and at 3, 6, and 12 months postoperatively. Baseline assessment was that measured on the admission day, 1 day prior to surgery. This study focuses on the results of laparoscopic RYGB, because this is the main procedure performed by our bariatric unit; the results of laparoscopic sleeve gastrectomy are described only briefly (Figure 2).

\section{Statistical analysis}

All continuous data are presented as the mean \pm standard deviation, and categorical data are presented as frequencies. Student's $t$-tests (paired and unpaired) were used to test the significance of differences between values for continuous

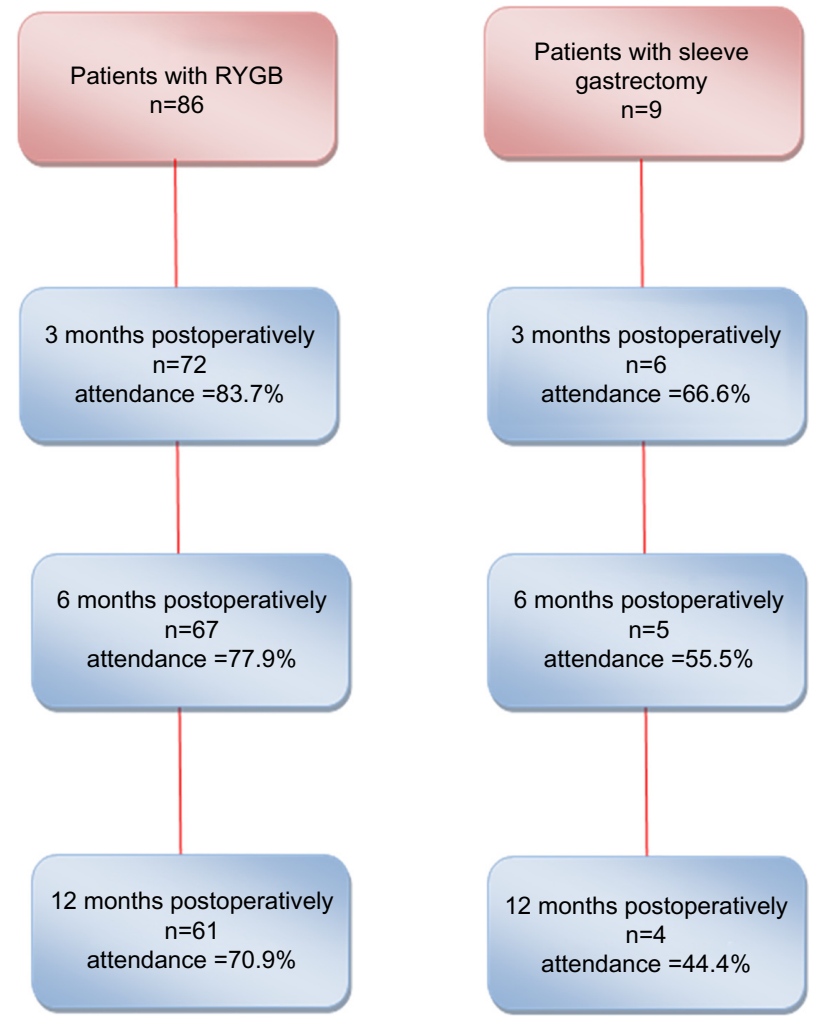

Figure 2 Patient follow-up flowchart for laparoscopic RYGB and laparoscopic sleeve gastrectomy, showing the numbers of patients who underwent each procedure at Rashid Center for Diabetes and Research and those with completed profiles at 3, 6 , and 12 months.

Notes: As demonstrated, laparoscopic RYGB was the main procedure performed by our bariatric unit. Patients who achieved more than $50 \%$ EBW at 12 months had significantly lower body mass index at baseline $(P<0.00 I)$.

Abbreviations: EBW, excessive body weight; RYGB, Roux-en-Y gastric bypass.

variables measured at baseline and at various time points. Differences with $P$-values $<0.05$ were considered to be statistically significant.

\section{Results}

\section{Laparoscopic RYGB}

Most patients $(90 \%, \mathrm{n}=86)$ underwent laparoscopic RYGB; of these, $72 \%$ were women $(n=62)$. Table 1 shows the baseline characteristics of the patients. Men had significantly

Table I Baseline characteristics of the studied RYGB patients

\begin{tabular}{ll}
\hline Variable & RYGB $^{\mathbf{a}}$ \\
\hline $\mathrm{n}$ & 86 \\
Age, years & $32.96 \pm 8.28$ \\
Sex, n (\%) & \\
$\quad$ Women & $62(72.09)$ \\
$\quad$ Men & $24(27.91)$ \\
Diabetes: no & $26(30.23)$ \\
\hline
\end{tabular}

Notes: ${ }^{a}$ Continuous data are presented as the mean \pm standard deviation, and categorical data are presented as the number and frequency.

Abbreviation: RYGB, Roux-en-Y gastric bypass. 
higher weight at baseline than women (137.68 \pm 15.88 and $117.51 \pm 15.64 \mathrm{~kg}$, respectively, $P<0.001)$. Sixty-one of these patients $(71 \%)$ had complete profiles at 12 months and 25 had incomplete profiles. At baseline, the weight of patients with complete profiles was significantly higher than that of those with incomplete profiles $(126.32 \pm 17.25$ and $110.39 \pm 16.38 \mathrm{~kg}$, respectively, $P<0.001)$, and the same was true of BMI $\left(47.04 \pm 6.04 \mathrm{~kg} / \mathrm{m}^{2}\right.$ and $41.83 \pm 5.11 \mathrm{~kg} /$ $\mathrm{m}^{2}$, respectively, $\left.P<0.01\right)$. Thirty percent $(\mathrm{n}=26)$ of patients had type 2 diabetes; among these, $73 \%(n=19)$ were women. No major complications (leakage, bleeding) were observed after surgery, with the exception of one patient who required reoperation to change sleeve gastrectomy to RYGB due to a corkscrew phenomenon secondary to anatomical predisposition (hanging stomach). The average length of stay in the hospital was 2-3 days. No deaths occurred postoperatively.

\section{Weight}

Patients had lost an average of $29.5 \%$ of their presurgery weight at 12 months after surgery (Table 2). Nine patients $(15 \%)$ achieved a BMI of less than $25 \mathrm{~kg} / \mathrm{m}^{2}$ by 12 months postoperatively. Baseline and 12-month postoperative weights $(126.32 \pm 17.39 \mathrm{~kg}$ and $87.21 \pm 15.24 \mathrm{~kg}$, respectively, $P<0.001)$ and BMI $\left(47.04 \pm 6.03 \mathrm{~kg} / \mathrm{m}^{2}\right.$ and $32.39 \pm 6.01 \mathrm{~kg} / \mathrm{m}^{2}$, respectively, $\left.P<0.001\right)$ differed significantly in patients with a complete profile at 12 months $(n=61)$. Men $(n=19)$ achieved significantly greater weight loss at 12 months compared with women $(\mathrm{n}=42 ; 46.44 \pm 14.76$ and $35.49 \pm 9.53$, respectively, $P<0.01$ ). No significant difference in weight loss was found between diabetic $(n=14)$ and nondiabetic $(n=47)$ patients at 12 months $(P=0.06)$.

\section{Percentage of excess weight loss}

By 12 months postoperatively, 77\% $(n=47)$ of patients had achieved more than 50\% EWL, compared with 51\% $(n=34)$ at 6 months (Figure 3$)$. Diabetic $(n=14)$ and nondiabetic $(\mathrm{n}=47)$ patients did not significantly differ in $\% \mathrm{EWL}$ $(P<0.18)$ at 12 months. Baseline BMI significantly differed
$(P<0.001)$ between patients who achieved EWL $>50 \%$ at 12 months $(n=48,45.82 \pm 5.25)$ and those who did not $(n=13,51.24 \pm 6.3$; Figure 1).

\section{Body composition}

The body component that decreased the most following surgery was fat mass. At 12 months, fat mass was reduced by $46 \%$, muscle mass was reduced by $11.64 \%$, and water mass was reduced by $12.68 \%$ (Figure 4 ). By 6 months, fat loss represented $75.48 \%$ of weight loss, and this remained the same even at 12 months $(78.95 \%)$. The contributions of fat-free mass to weight loss were $24.52 \%$ and $21.05 \%$ at 6 and 12 months, respectively. Fat mass differed significantly between baseline $(58.1 \pm 10.74 \mathrm{~kg})$ and 12 months $(31.95 \pm 13.71 \mathrm{~kg})$ for patients with complete profiles $(\mathrm{n}=61$, $P<0.001)$. At 12 months, men $(\mathrm{n}=19)$ had achieved significantly higher fat mass loss than women $(\mathrm{n}=42 ; 35.59 \pm 11.75 \mathrm{~kg}$ and $24.3 \pm 10.12 \mathrm{~kg}$, respectively, $P<0.01)$. However, women lost significantly greater water mass than men $(6.51 \pm 3.93 \mathrm{~kg}$ and $3.96 \pm 4.05 \mathrm{~kg}$, respectively, $P=0.04$ ).

\section{Metabolic marker outcomes}

Systolic and diastolic blood pressure decreased by $3.8 \%$ and $2.7 \%$, respectively (Table 3 ). However, the decrease in diastolic blood pressure was not significant compared with baseline for patients with complete 12-month profiles $(P=0.21)$. All lipid profile measures (low-density lipoprotein, triglycerides, and total cholesterol) were significantly reduced $(P<0.01)$ at 12 months compared with baseline; triglyceride levels showed the greatest mean improvement (26.71\%), followed by low-density lipoprotein $(20.9 \%)$ and total cholesterol (12.26\%). High-density lipoprotein increased significantly $(25 \%)$ at 12 months $(P<0.001)$. The liver profile measures of gamma-glutamyl transferase, alanine transaminase, and aspartate transaminase decreased by $50.54 \%$, $39.83 \%$, and $15.41 \%$, respectively, and these decreases were significant at 12 months $(P<0.05)$. Alkaline phosphatase slightly decreased (4.6\%) but was not significantly different

Table 2 Weight outcomes for RYGB

\begin{tabular}{|c|c|c|c|c|}
\hline \multirow[t]{2}{*}{ Variable } & \multicolumn{4}{|l|}{ RYGBa } \\
\hline & Baseline $(n=86)$ & 3 months $(n=72)$ & 6 months $(n=67)$ & 12 months $(n=6 I)$ \\
\hline Weight (kg) & $123.67 \pm 18.03$ & $99.96 \pm 13.83^{b}$ & $96.31 \pm 15.54^{b}$ & $87.43 \pm 15.18^{b}$ \\
\hline Weight loss (kg) & - & $26.74 \pm 13.86$ & $32.59 \pm 19.68$ & $47.04 \pm 25.5$ \\
\hline $\mathrm{BMI}\left(\mathrm{kg} / \mathrm{m}^{2}\right)$ & $45.99 \pm 6.2 \mathrm{I}^{\mathrm{b}}$ & $4 I .45 \pm 5.7 I^{b}$ & $36.53 \pm 5.54^{b}$ & $32.42 \pm 5.93^{b}$ \\
\hline BMI decrease $\left(\mathrm{kg} / \mathrm{m}^{2}\right)$ & - & $7.69 \pm 5.18$ & $12.22 \pm 8.07$ & $17.85 \pm 9.9$ \\
\hline
\end{tabular}

Notes: a Data are presented as the mean \pm standard deviation; bsignificantly different from baseline according to a paired $t$-test $(P<0.000)$. Abbreviations: BMI, body mass index; RYGB, Roux-en-Y gastric bypass. 


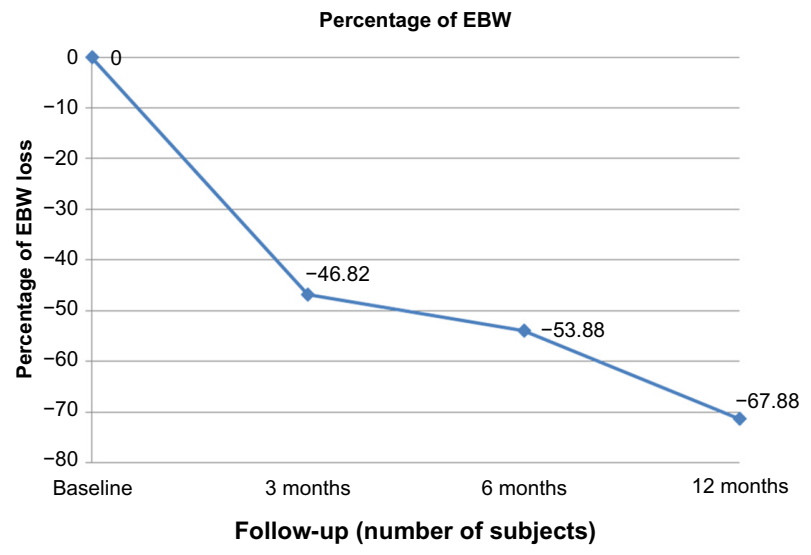

Figure 3 Excess body weight loss at various times after the laparoscopic RYGB procedure.

Notes: At 12 months, the average \% EBW loss was approximately $68 \%$ for 61 patients with completed profiles. Approximately $77 \%$ of patients $(n=47)$ lost more than $50 \%$ of EBW at 12 months compared with $51 \%(n=34)$ at 6 months. Baseline body mass index $(P<0.00 \mathrm{I})$ significantly differed between patients who achieved an EBW loss $>50 \%$ at 12 months $\left(n=48,45.82 \pm 5.25 \mathrm{~kg} / \mathrm{m}^{2}\right)$ and those who did not $\left(n=13,5 \mid .24 \pm 6.3 \mathrm{~kg} / \mathrm{m}^{2}\right)$.

Abbreviations: EBW, excessive body weight; RYGB, Roux-en-Y gastric bypass.

from baseline $(P=0.08)$. Albumin also slightly decreased $(2.47 \%)$ but not significantly ( $P=0.25)$. No significant difference was found between diabetics $(n=14)$ and nondiabetics $(\mathrm{n}=47)$ for any variable except fasting blood glucose and $\mathrm{HbA}_{\mathrm{lc}}(P=0.02$ and $P=0.004$, respectively).
At the 12-month visit, fasting blood glucose, $\mathrm{HbA}_{1 \mathrm{c}}$, and insulin levels had dramatically decreased by $66.35 \%, 73.37 \%$, and $67.47 \%$, respectively, for type 2 diabetic patients. This reduction was statistically significant $(P<0.001)$ between baseline and 12 months for patients with complete profiles $(\mathrm{n}=15$; Figure 5). At 6 months postoperatively, diabetic patients had dramatically reduced their use of antidiabetic medications (Figure 6). The use of biguanides (metformin) and sulfonylureas (gliclazide) was reduced by $89.2 \%$ and $88.88 \%$, respectively. GLP-1 agonist (exenatide) and insulin use were completely stopped. Additionally, both the use of antihypertensive (angiotensin receptor blocker) and cholesterol-reducing (statin) medications decreased by $90 \%$. By 6 months, $37 \%$ of patients $(n=7)$ had achieved complete remission of type 2 diabetes, and by 12 months, $43 \%$ of patients $(n=6)$ were cured of type 2 diabetes.

\section{Sleeve gastrectomy}

Eight patients underwent laparoscopic sleeve gastrectomy, of whom six were women (75\%) and two were diabetics (25\%). Four subjects had complete profiles at 12 months. Patients who underwent laparoscopic sleeve gastrectomy lost approximately $22.7 \%$ of their total weight and an average of $61.33 \%$

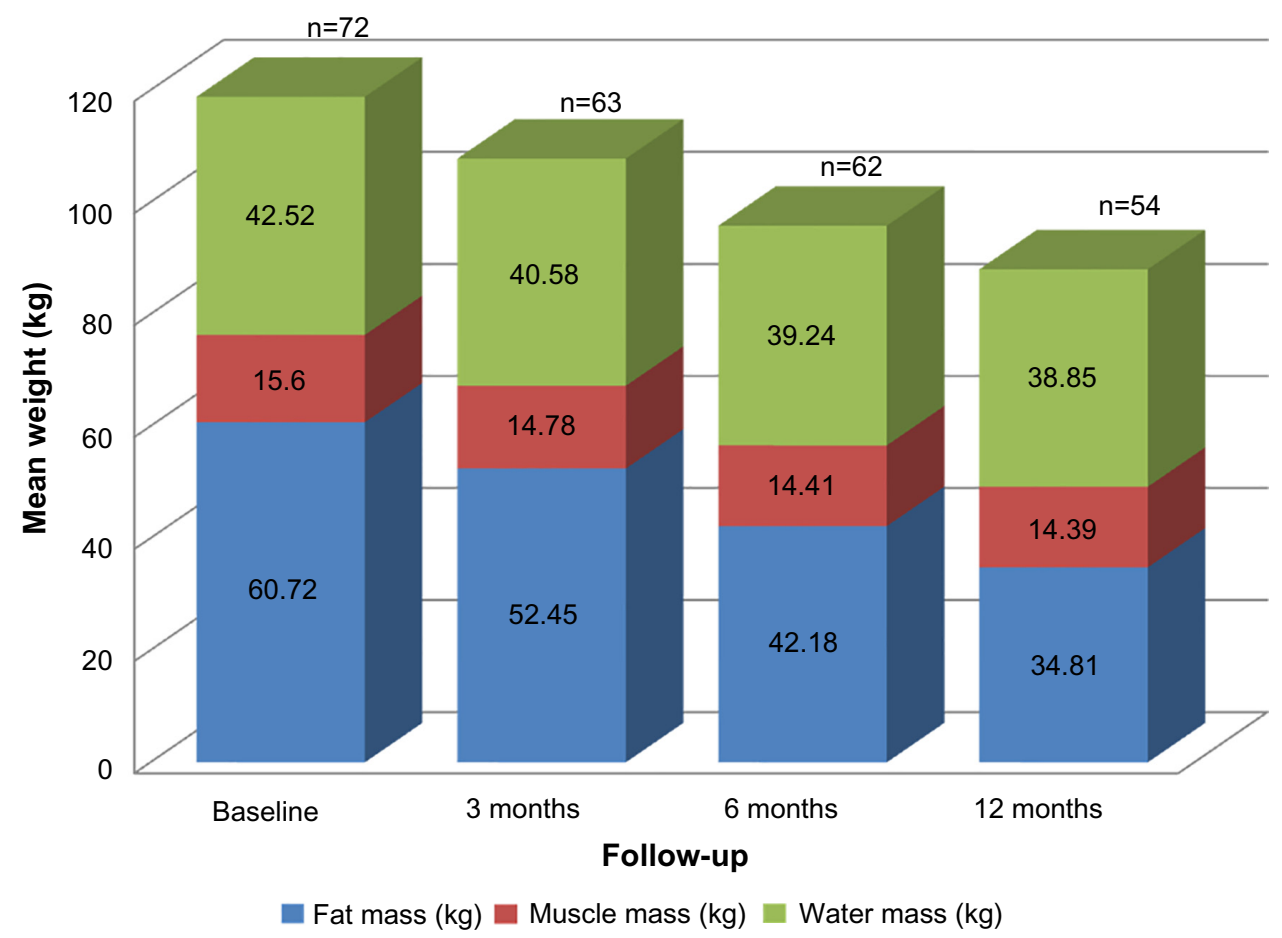

Figure 4 Changes in body composition at various times after laparoscopic RYGB surgery.

Notes: Fat mass decreased the most postoperatively. At 12 months, patients had achieved average reductions of $46 \%$ in fat mass, $11.64 \%$ in muscle mass, and I $2.68 \%$ in water mass. Fat mass differed significantly between baseline $(58.1 \pm 10.74 \mathrm{~kg})$ and 12 months $(31.95 \pm 13.71 \mathrm{~kg})$ for patients with complete profiles $(\mathrm{n}=54$, $P<0.00 \mathrm{I})$. At $12 \mathrm{months}$, the 19 men achieved significantly greater fat mass loss than the 42 women $(35.59 \pm \mathrm{I} I .75 \mathrm{~kg}$ versus $24.3 \pm 10.12 \mathrm{~kg}, P<0.0 \mathrm{I})$.

Abbreviation: RYGB, Roux-en-Y gastric bypass. 
Table 3 Changes in metabolic markers outcomes post RYGB

\begin{tabular}{|c|c|c|c|c|}
\hline \multirow[t]{2}{*}{ Variable } & \multicolumn{4}{|l|}{ RYGB $^{\mathbf{a}}$} \\
\hline & Baseline $(n=86)$ & 3 months $(n=72)$ & 6 months $(n=67)$ & 12 months $(n=6 I)$ \\
\hline $\mathrm{HbA}_{\mathrm{Ic}}(\%)^{\mathrm{b}}$ & $5.43 \pm 0.4$ & $5.54 \pm 0.74$ & $5.35 \pm 0.92$ & $5.33 \pm 0.43$ \\
\hline Fasting blood glucose (mmol/L) ${ }^{\mathrm{b}}$ & $8.85 \pm 3.36$ & $5.34 \pm 1.27$ & $5.04 \pm 0.42$ & $4.96(0.5)$ \\
\hline Insulin $(\mu \mathrm{U} / \mathrm{mL})$ & $25.61 \pm 15.64$ & $15.39 \pm 9.15^{c}$ & $10.86 \pm 8.00^{c}$ & $8.33 \pm 2.78^{c}$ \\
\hline \multicolumn{5}{|l|}{ Blood pressure (mmHg) } \\
\hline Systolic & $128.36 \pm 14.16$ & $123.33 \pm 11.59^{c}$ & $|20.07 \pm 1| .62^{c}$ & $123.45 \pm 13.10^{c}$ \\
\hline Diastolic & $72.34 \pm 10.96$ & $70.56 \pm 9.12$ & $69.69 \pm 9.29$ & $69.66 \pm 9.21$ \\
\hline \multicolumn{5}{|l|}{ Lipid profile } \\
\hline $\mathrm{LDL}(\mathrm{mmol} / \mathrm{L})$ & $3.0 I \pm 0.67$ & $22.99 \pm 9.93^{c}$ & $2.52 \pm 0.62^{c}$ & $2.38 \pm 0.68^{c}$ \\
\hline $\mathrm{HDL}(\mathrm{mmol} / \mathrm{L})$ & $1.20 \pm 1.2$ & $3.73 \pm 2.25^{c}$ & $1.34 \pm 0.38^{c}$ & $1.5 \pm 0.38^{c}$ \\
\hline Triglycerides (mmol/L) & $|.31 \pm 0.6|$ & $1.33 \pm 0.39^{c}$ & $0.95 \pm 0.33^{c}$ & $0.96 \pm 0.75^{c}$ \\
\hline Total cholesterol (mmol/L) & $4.73 \pm 0.88$ & $9.26 \pm 4.70^{c}$ & $4.26 \pm 0.73^{c}$ & $4.15 \pm 0.86^{c}$ \\
\hline \multicolumn{5}{|l|}{ Liver profile } \\
\hline ALP (IU/L) & $74.84 \pm 20.65$ & $64.92 \pm 19.92^{c}$ & $71.01 \pm 21.03$ & $71.4 \pm 23.32$ \\
\hline ALT (IU/L) & $27.44 \pm 20.93$ & $32.36 \pm 23.15^{c}$ & $18.75 \pm \mid 1.2^{c}$ & $16.51 \pm 7.6^{c}$ \\
\hline AST (U/L) & $20.5 I \pm 8.43$ & $23.14 \pm 10.04^{c}$ & $17.35 \pm 6.97^{c}$ & $17.11 \pm 5.99^{c}$ \\
\hline GGT (IU/L) & $29.84 \pm 16.52$ & $11.96 \pm 7.2 I^{c}$ & $15.66 \pm 7.4^{c}$ & $14.76 \pm 6.45^{c}$ \\
\hline Albumin $(g / L)$ & $40.89 \pm 2.87$ & $40.37 \pm 3.7$ & $40.69 \pm 2.67$ & $41.9 \pm 8.28$ \\
\hline
\end{tabular}

Notes: a Data are presented as the mean \pm standard deviation; ${ }^{b}$ values are presented for nondiabetic patients; ${ }^{c}$ significantly different from baseline according to a paired $t$-test $(P<0.05)$.

Abbreviations: ALP, alkaline phosphatase; ALT, alanine transaminase; AST, aspartate transaminase; GGT, gamma-glutamyl transferase; HDL, high-density lipoprotein; LDL, low-density lipoprotein; RYGB, Roux-en-Y gastric bypass; $\mathrm{HbA}_{\mathrm{Ic}}$, glycated hemoglobin.

$(n=4)$ of excess body weight at 12 months. At 12 months after surgery, fat, muscle, and water mass decreased by $49.36 \%$, $9.85 \%$, and $9.84 \%$, respectively. All laboratory test values were altered after sleeve gastrectomy. Triglycerides, lowdensity lipoprotein, and total cholesterol were reduced by $42.75 \%, 11.25 \%$, and $8.2 \%$, respectively, and high-density lipoprotein increased by $17.52 \%$. Regarding liver enzymes, alanine transaminase, aspartate transaminase, and alkaline phosphatase were reduced by $50 \%, 24.61 \%$, and $14.29 \%$, respectively; gamma-glutamyl transferase was increased by $10.07 \%$. No change was observed in albumin levels after surgery $(0.37 \%)$.

\section{Discussion}

This retrospective study reported the short-term outcomes (weight and metabolic parameters) of bariatric surgery (focusing on laparoscopic RYGB) in Emirati adults. Although laparoscopic RYGB is one of the most commonly performed bariatric procedures used to treat severe obesity in the UAE, few data have been published regarding its outcome in Emiratis. Our study demonstrated the short-term positive impact of laparoscopic RYGB surgery on weight, body composition, and comorbidities, including lipid profiles. Likewise, glucose control was dramatically improved in patients with type 2 diabetes, and the use of antidiabetic medications decreased accordingly. Additionally, no evidence of major postoperative complications was found.
These positive outcomes of RYGB surgery are attributable to a complex interaction between several mechanisms. RYGB causes weight loss mainly through gastric restriction and surgical malabsorption. ${ }^{16}$ It has also been suggested that the surgery affects eating behavior. Patients normally experience appetite changes and reduced hunger after surgery, possibly due to changes in appetite and in the levels of satiety hormones including ghrelin, glucose-dependent insulinotropic peptide, GLP-1, and peptide YY. ${ }^{17}$ Moreover, patients might be cautious in their food selection and in the quantities consumed to avoid unpleasant symptoms associated with dumping syndrome (vomiting, nausea, abdominal discomfort) or when exceeding the capacity of their small gastric pouch. ${ }^{16}$ However, the dramatic improvement seen in glycemic control in type 2 diabetic patients after surgery is said to be independent of the treatment of obesity and is not entirely explained by postoperative weight loss and restriction of calorie intake. ${ }^{18}$ Alterations in the anatomy of the gastrointestinal tract and neural and hormonal changes (including changes in incretins) can partly explain the improvement in glycemic control after surgery; bariatric procedures such as laparoscopic RYGB can be considered an effective treatment strategy for type 2 diabetes. ${ }^{18,19}$ This concept was not assessed while developing the outcome measures in our bariatric program.

Despite the increased use of bariatric surgery in the UAE, published data on its outcomes in this population are lacking. ${ }^{10}$ 


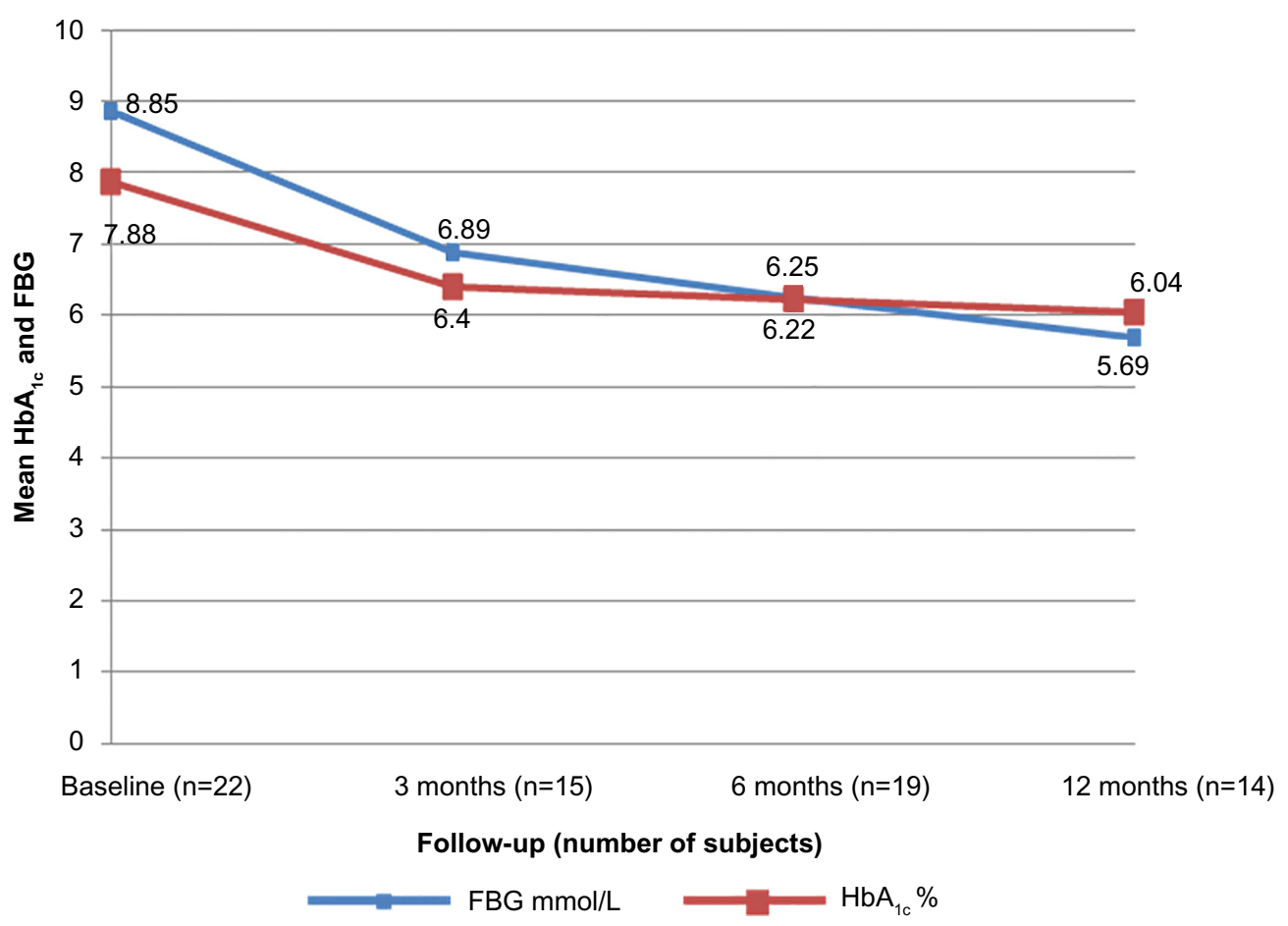

Figure 5 Postoperative changes in mean $\mathrm{HbA}_{\mathrm{Ic}}$ and $\mathrm{FBG}$ in patients with type 2 diabetes at various times. $\mathrm{Hb} \mathrm{A}_{\mathrm{lc}}$ and $\mathrm{FBG}$ levels both decreased significantly $(P<0.005)$ at 6 and 12 months.

Abbreviations: FBG, fasting blood glucose; $\mathrm{HbA}_{1 \mathrm{c}}$, glycated hemoglobin.

The baseline demographics of the population studied here are similar to those reported internationally in terms of BMI $\left(45.99 \mathrm{~kg} / \mathrm{m}^{2}\right)$ and sex (71\% women), and the patients studied were younger (32.96 years) than those included in a meta-analysis on bariatric outcome which had 136 studies (BMI $46.85 \mathrm{~kg} / \mathrm{m}^{2}, 72.6 \%$ women, mean age 38.97 years). ${ }^{20}$ However, the $68 \%$ EWL found here was higher than the average $\%$ EWL reported by the latter review $(61.56 \%, n=4,204)$ for the RYGB procedure. ${ }^{20}$ This might be due to their inclusion of both short-term ( $<2$ years) and long-term $(>2$ years) outcomes, whereas our study reports results covering a maximum of 12 months. Looking at similar short follow-up studies, our results fell within the wide range observed. Percentage EWLs of $51.3 \%(n=24), 60.5 \%(n=16)$, and $64.3 \%(n=111),{ }^{21}$ and $86 \%(\mathrm{n}=75)^{22}$ were reported after laparoscopic RYGB.

Other important findings of our study included the changes in body composition that followed the laparoscopic RYGB procedure. One of the main goals of any weight loss intervention is to reduce excessive body fat mass, which is related to metabolic disorders, and also to minimize the loss of fat-free mass that might be undesirable, although inevitable, with drastic weight loss interventions. ${ }^{23}$ In our study, we reported a fat mass loss of $46 \% 12$ months after RYGB, a value that is similar to those observed by others, including Ciangura et $\mathrm{al}^{24}(45.2 \%, \mathrm{n}=42)$ and Tamboli et $\mathrm{al}^{25}$ $(50 \%, n=29)$.

In terms of the contribution of fat-free mass to body weight loss, our study reported similar fat-free mass loss to that reported by Das et $\mathrm{al}^{26}$ ( $21 \%$ of total weight loss) and a lower loss of fat-free mass than that reported by Ciangura et $\mathrm{al}^{24}$ ( $27 \%$ of total weight loss) or by Chaston et $\mathrm{al}^{23}$ (an average of $31.3 \%$ of total weight loss); the time points were not consistent among the studies included in the latter review.

Consensus is lacking in the literature regarding the level of loss of fat-free mass that is considered excessive in terms of undesirable metabolic outcome. ${ }^{23}$ However, the percentage of fat-free mass loss observed in our study was not associated with significant changes in post-surgery albumin levels, an indicator of clinical malnutrition. To minimize loss of fat-free mass after surgery, all of our patients underwent frequent follow-up visits with the clinical dietitian, following the post-bariatric surgery protocol; during these visits, recommendations concerning the importance of a well-balanced diet and sufficient protein were routinely reinforced.

The changes in comorbidity parameters observed here are consistent with the findings of many studies which document an improved lipid profile post-RYGB surgery, either through decreased total serum cholesterol, triglyceride, and low- 


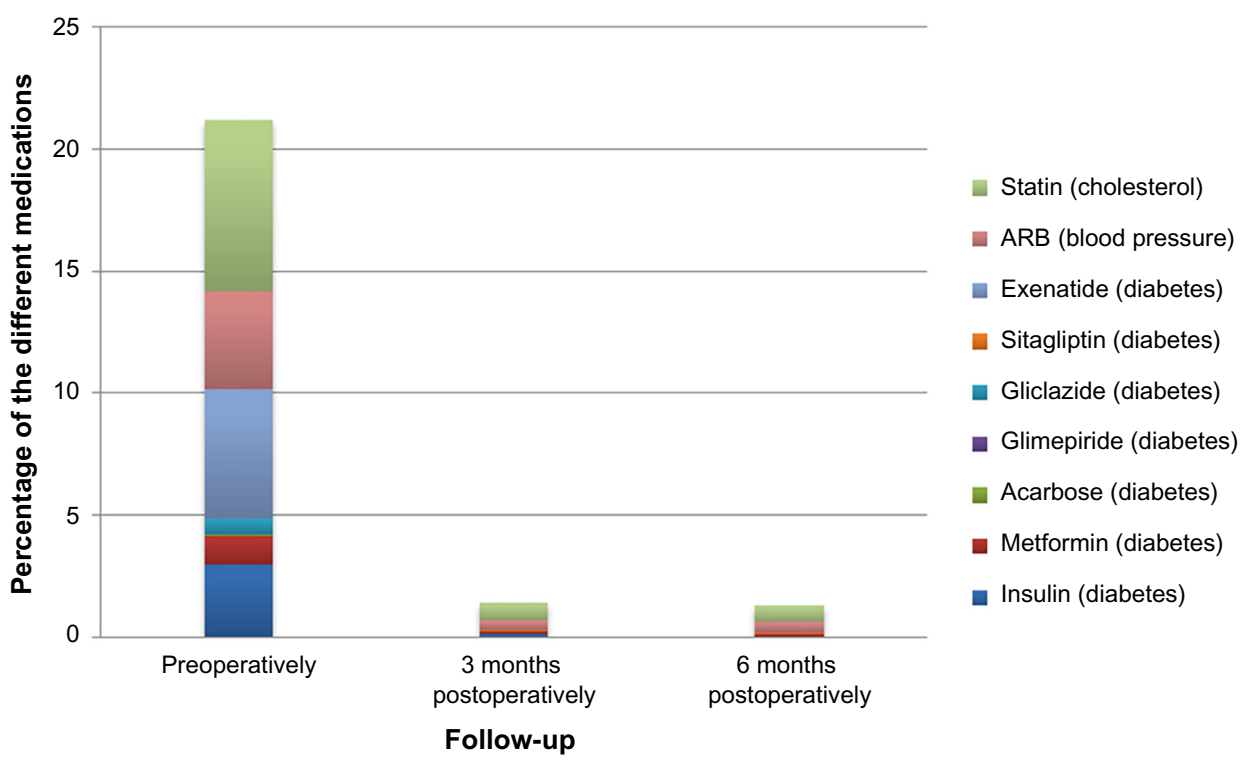

Figure 6 Changes in medication used by patients with diabetes after RYGB.

Notes: At 6 months postoperatively, the use of antidiabetic medications had dramatically decreased. The use of biguanides (metformin) and sulfonylureas (gliclazide) decreased by $89.2 \%$ and $88.88 \%$, respectively. Glucagon-like peptide-I agonists (exenatide) and insulin were stopped completely. Additionally, use of antihypertensive (ARB) and cholesterol-lowering (statin) medications both decreased by $90 \%$.

Abbreviations: ARB, angiotensin receptor blocker; RYGB, Roux-en-Y gastric bypass.

density lipoprotein levels or through increased high-density lipoprotein levels. ${ }^{27,28}$ Improvements in the lipid profile arguably reduce cardiovascular risk after surgery. Indeed, such improvements were considered to significantly reduce 10-year cardiovascular risk according to the Framingham risk equation from $6.7 \%$ to $5.4 \%$ at the 12 -month follow-up postRYGB surgery. ${ }^{29}$ Similarly, for type 2 diabetes, our results demonstrated a complete remission rate of $39 \%$ by 12 months after surgery and a dramatic improvement $(74 \%)$ in $\mathrm{HbA}_{1 \mathrm{c}}$. Our results are consistent with those of Pournaras et al, ${ }^{30}$ who reported a $40.6 \%$ remission in type 2 diabetes $(n=160)$ post gastric bypass surgery in three bariatric centers.

Several studies have used different remission/resolution definitions for type 2 diabetes, possibly contributing to the variance in rates observed in the literature. We used the American Diabetes Association definition of complete remission of type 2 diabetes, ${ }^{14}$ which can yield lower remission rates. For instance, Hall et $\mathrm{al}^{31}$ reported a higher rate of type 2 diabetes resolution $(68.4 \%, n=67)$, whereas Mingrone et $\mathrm{al}^{32}$ observed $75 \%(\mathrm{n}=20)$ remission after RYGB. Despite these differences and due to the dramatic improvement in glycemic control observed here, our results add to the evidence supporting use of laparoscopic RYGB as an effective treatment for obese patients with type 2 diabetes (based on results obtained shortly after the operation) that can drastically decrease the need for antidiabetic medication. The elevated aspartate transaminase and alanine transaminase levels observed at baseline in our patients might indicate a history of liver abnormalities, particularly nonalcoholic fatty liver disease, for which a significant reduction has been demonstrated post-surgery. However, the use of liver test values for the diagnosis of liver disease has limitations and can be considered a poor predictive diagnostic tool. ${ }^{33}$ We also observed reductions in systolic and diastolic blood pressures, consistent with other reports that describe reductions in blood pressure post-RYGB; ${ }^{32,34}$ however, diastolic blood pressure was not significantly reduced. One reason for the latter finding might be that the average diastolic blood pressure was within the normal range at baseline.

Collectively, the results of bariatric surgery observed at this center, particularly those for laparoscopic RYGB, achieved dramatic weight loss and improved metabolic markers in the short term. Our results also replicate bariatric surgery results observed internationally.

Considering the economic burden of obesity and its comorbidities on the Emirati health care system, bariatric surgery is an increasingly attractive treatment for obesity and type 2 diabetes due to its ability to reduce associated health care costs. Indeed, it is evident from the literature that bariatric surgery is superior to conventional weight loss treatment in terms of reducing health care costs, increasing associated health benefits, and adding to patients' quality of life. ${ }^{35,36}$ More particularly, laparoscopic RYGB, the main 
bariatric surgery performed at our center, is superior to other procedures (such as gastroplasty, jejunoileal bypass, and open RYGB) in terms of the occurrence of complications, weight loss, and comorbidity outcomes. ${ }^{37}$

\section{Limitations}

One of the main limitations of this study is its retrospective design; such designs are associated with selection bias due to a lack of randomization. Furthermore, potential confounders were not properly accounted for due to the study type and the homogeneity of the subjects. Additionally, the data were retrieved retrospectively; therefore, inaccuracies in data collection were inevitable. However, our data were collected in an evidence-based bariatric program by trained personnel, possibly increasing the accuracy of the data entries. Sleeve gastrectomy was also performed; however, due to the small number of patients who underwent this procedure, we were not able to elaborate on its results or compare it with laparoscopic RYGB outcomes.

Our results were similar to international outcomes in the short term; however, collection of long-term data is warranted to determine whether Emiratis maintain a trend similar to that shown in other countries (international data show maximum weight loss at 1-2 years after surgery and a subsequent increase in weight in the following years, although the weight gain has been reported to level off after $8-10$ years). ${ }^{8}$ Despite the positive results following from bariatric surgery and its advantages over conventional treatment, it is important to mention that surgery cannot be offered to all patients who suffer from obesity. Hence, nonsurgical treatment involving dietary intervention, physical activity, and behavioral therapy should remain the first line of treatment for severely obese patients. Nevertheless, reporting on bariatric outcomes and studying the physiological changes caused by surgery might lead to better treatments of obesity and type 2 diabetes.

\section{Acknowledgments}

The authors would like to acknowledge the adjacent Sheikh Khalifa Hospital for their collaboration in performing the surgeries. They also thank the Global Health Partner Diabetes Center UAE for their support in establishing the bariatric clinic.

\section{Author contributions}

Salah Abusnana, Sarah Abdi, and Almantas Maleckas participated in the design of the study. Salah Abusnana, Sarah Abdi, Brigette Tagure, Murtada Elbagir, and Almantas Maleckas collected the data. Salah Abusnana and Sarah Abdi analyzed the data. Salah Abusnana and Sarah Abdi wrote the paper. Salah Abusnana and Almantas Maleckas had the primary responsibility for the final outcome and provided overall supervision. All authors made substantial contributions to conception and design, acquisition of data, or analysis and interpretation of data; took part in either drafting the article or revising it critically for important intellectual content; gave final approval of the version to be published; and agree to be accountable for all aspects of the work.

\section{Disclosure}

The authors report no conflicts of interest in this work.

\section{References}

1. World Health Organization. Health topics: obesity. Geneva, Switzerland: World Health Organization; 2014. Available from: http://www.who.int/ topics/obesity/en/. Accessed July 28, 2015.

2. Emro.who.int [Internet]. World Health Organization Regional Office for the Eastern Mediterranean, Obesity. Cairo, Egypt; 2014. Available from http://www.emro.who.int/health-topics/obesity/. Accessed August 17, 2015.

3. World Health Organization Regional Office for the Eastern Mediterranean. Country cooperation strategy for WHO and United Arab Emirates 2005-2009. Cairo, Egypt: EMRO; 2006. Available from: http://www.who.int/countryfocus/cooperation_strategy/countries/ ccs_final_are_2005_en.pdf. Accessed August 17, 2015.

4. Dee A, Kearns K, O'Neill C, et al. The direct and indirect costs of both overweight and obesity: a systematic review. BMC Res Notes. 2014;7:242.

5. Al-Maskari F, El-Sadig M, Nagelkerke N. Assessment of the direct medical costs of diabetes mellitus and its complications in the United Arab Emirates. BMC Public Health. 2010;10:679.

6. National Heart Lung and Blood Institute. The practical guide: identification, evaluation, and treatment of overweight and obesity in adults. Bethesda, MD, USA: National Heart Lung and Blood Institute; 2000. Available from: http://www.nhlbi.nih.gov/files/docs/guidelines/ prctgd_c.pdf. Accessed July 28, 2015.

7. Gloy VL, Briel M, Bhatt DL, et al. Bariatric surgery versus non-surgical treatment for obesity: a systematic review and meta-analysis of randomised controlled trials. BMJ. 2013;347:f5934.

8. Sjöström L, Narbro K, Sjöström CD, et al. Effects of bariatric surgery on mortality in Swedish obese subjects. N Engl J Med. 2007;357: 741-752.

9. Buchwald H, Oien DM. Metabolic/bariatric surgery worldwide 2008. Obes Surg. 2009;19:1605-1611.

10. Buchwald H, Oien DM. Metabolic/bariatric surgery worldwide 2011. Obes Surg. 2013;23(4):427-436.

11. Nimeri A, Mohamed A, El Hassan E, et al. Are results of bariatric surgery different in the Middle East? Early experience of an international bariatric surgery program and an ACS NSQIP outcomes comparison. J Am Coll Surg. 2013;216:1082-1088.

12. Admiraal WM, Celik F, Gerdes VE, Dallal RM, Hoekstra JB, Holleman F. Ethnic differences in weight loss and diabetes remission after bariatric surgery: a meta-analysis. Diabetes Care. 2012;35: 1951-1958.

13. Mechanick JI, Youdim A, Jones DB, et al. Clinical practice guidelines for the perioperative nutritional, metabolic, and nonsurgical support of the bariatric surgery patient - 2013 update: cosponsored by American Association of Clinical Endocrinologists, The Obesity Society, and American Society for Metabolic and Bariatric Surgery. Obesity (Silver Spring). 2013;21 Suppl 1:S1-S27.

14. Biesemeier C, Garland J. ADA Pocket Guide to Bariatric Surgery. Chicago, IL, USA: American Dietetic Association; 2009. 
15. Buse JB, Caprio S, Cefalu WT, et al. How do we define cure of diabetes? Diabetes Care. 2009;32:2133-2135.

16. Elder KA, Wolfe BM. Bariatric surgery: a review of procedures and outcomes. Gastroenterology. 2007;132:2253-2271.

17. Beckman LM, Beckman TR, Earthman CP. Changes in gastrointestinal hormones and leptin after Roux-en-Y gastric bypass procedure: a review. J Am Diet Assoc. 2010;110:571-584.

18. Rubino F. Is type 2 diabetes an operable intestinal disease? A provocative yet reasonable hypothesis. Diabetes Care. 2008;31 Suppl 2: S290-S296.

19. Rubino F, R'bibo SL, del Genio F, Mazumdar M, McGraw TE. Metabolic surgery: the role of the gastrointestinal tract in diabetes mellitus. Nat Rev Endocrinol. 2010;6:102-109.

20. Buchwald H, Avidor Y, Braunwald E, et al. Bariatric surgery: a systematic review and meta-analysis. JAMA. 2004;292:1724-1737.

21. Franco JV, Ruiz PA, Palermo M, Gagner M. A review of studies comparing three laparoscopic procedures in bariatric surgery: sleeve gastrectomy, Roux-en-Y gastric bypass and adjustable gastric banding. Obes Surg. 2011;21:1458-1468.

22. Lakdawala MA, Bhasker A, Mulchandani D, Goel S, Jain S. Comparison between the results of laparoscopic sleeve gastrectomy and laparoscopic Roux-en-Y gastric bypass in the Indian population: a retrospective 1 year study. Obes Surg. 2010;20:1-6.

23. Chaston TB, Dixon JB, O'Brien PE. Changes in fat-free mass during significant weight loss: a systematic review. Int J Obes (Lond). 2007;31: 743-750.

24. Ciangura C, Bouillot JL, Lloret-Linares C, et al. Dynamics of change in total and regional body composition after gastric bypass in obese patients. Obesity (Silver Spring). 2010;18:760-765.

25. Tamboli RA, Hossain HA, Marks PA, et al. Body composition and energy metabolism following Roux-en-Y gastric bypass surgery. Obesity (Silver Spring). 2010;18:1718-1724.

26. Das SK, Roberts SB, McCrory MA, et al. Long-term changes in energy expenditure and body composition after massive weight loss induced by gastric bypass surgery. Am J Clin Nutr. 2003;78:22-30.
27. Nguyen NT, Varela E, Sabio A, Tran CL, Stamos M, Wilson SE. Resolution of hyperlipidemia after laparoscopic Roux-en-Y gastric bypass. J Am Coll Surg. 2006;203:24-29.

28. Jamal M, Wegner R, Heitshusen D, Liao J, Samuel I. Resolution of hyperlipidemia follows surgical weight loss in patients undergoing Roux-en-Y gastric bypass surgery: a 6-year analysis of data. Surg Obes Relat Dis. 2011;7:473-479.

29. Arterburn D, Schauer DP, Wise RE, et al. Change in predicted 10-year cardiovascular risk following laparoscopic Roux-en-Y gastric bypass surgery. Obes Surg. 2009;19:184-189.

30. Pournaras DJ, Aasheim ET, Søvik TT, et al. Effect of the definition of type II diabetes remission in the evaluation of bariatric surgery for metabolic disorders. Br J Surg. 2012;99:100-103.

31. Hall TC, Pellen MG, Sedman PC, Jain PK. Preoperative factors predicting remission of type 2 diabetes mellitus after Roux-en-Y gastric bypass surgery for obesity. Obes Surg. 2010;20:1245-1250.

32. Mingrone G, Panunzi S, De Gaetano A, et al. Bariatric surgery versus conventional medical therapy for type 2 diabetes. $N \mathrm{Engl} \mathrm{J} \mathrm{Med.}$ 2012;366:1577-1585.

33. Alba LM, Lindor K. Review article: non-alcoholic fatty liver disease. Aliment Pharmacol Ther. 2003;17:977-986.

34. Ahmed AR, Rickards G, Coniglio D, et al. Laparoscopic Roux-en-Y gastric bypass and its early effect on blood pressure. Obes Surg. 2009; $19: 845-849$.

35. Hoerger TJ, Zhang P, Segel JE, Kahn HS, Barker LE, Couper S Cost-effectiveness of bariatric surgery for severely obese adults with diabetes. Diabetes Care. 2010;33:1933-1939.

36. Keating CL, Dixon JB, Moodie ML, et al. Cost-effectiveness of surgically induced weight loss for the management of type 2 diabetes: modeled lifetime analysis. Diabetes Care. 2009;32:567-574.

37. Clegg A, Colquitt J, Sidhu M, Royle P, Walker A. Clinical and cost effectiveness of surgery for morbid obesity: a systematic review and economic evaluation. Int J Obes Relat Metab Disord. 2003;27: 1167-1177.

\section{Publish your work in this journal}

Diabetes, Metabolic Syndrome and Obesity: Targets and Therapy is an international, peer-reviewed open-access journal committed to the rapid publication of the latest laboratory and clinical findings in the fields of diabetes, metabolic syndrome and obesity research. Original research, review, case reports, hypothesis formation, expert opinion and commentaries are all considered for publication. The manuscript management system is completely online and includes a very quick and fair peer-review system, which is all easy to use. Visit http://www.dovepress.com/testimonials.php to read real quotes from published authors. 